\title{
GENERATING RELATIONS INVOLVING 3-VARIABLE 2-PARAMETER TRICOMI FUNCTIONS USING LIE-ALGEBRAIC TECHNIQUES
}

\author{
Subuhi Khan, Mumtaz Ahmad Khan, and Rehana Khan
}

\begin{abstract}
This paper is an attempt to stress the usefulness of the multivariable special functions. In this paper, we derive generating relations involving 3-variable 2-parameter Tricomi functions by using Lie-algebraic techniques. Further we derive certain new and known generating relations involving other forms of Tricomi and Bessel functions as applications.
\end{abstract}

\section{Introduction}

The function

$$
C_{n}(x)=\sum_{r=0}^{\infty} \frac{(-1)^{r} x^{r}}{r !(n+r) !},
$$

is a Bessel like function known as Tricomi function and is characterized by the following link with the ordinary Bessel function $J_{n}(x)$ [11]:

$$
C_{n}(x)=x^{-n / 2} J_{n}(2 \sqrt{x})
$$

or

$$
J_{n}(x)=\left(\frac{x}{2}\right)^{n} C_{n}\left(\frac{x^{2}}{4}\right) .
$$

The Tricomi function $C_{n}(x)$ satisfies the generating function

$$
\exp \left(t-\frac{x}{t}\right)=\sum_{n=-\infty}^{\infty} C_{n}(x) t^{n}
$$

Received April 21, 2008.

2000 Mathematics Subject Classification. 33C10, 33C80, 33E20.

Key words and phrases. generalized Tricomi functions, Lie-algebra representation, generating relations.

This work has been done under a Major Research Project No. F.33-110/2007 (SR) sanctioned to the first author by the University Grants Commission, Government of India, New Delhi.

(C)2009 The Korean Mathematical Society 
which yield the recurrences

$$
\begin{aligned}
& \frac{d}{d x} C_{n}(x)=-C_{n+1}(x), \\
& x C_{n+1}(x)-n C_{n}(x)+C_{n-1}(x)=0 .
\end{aligned}
$$

On combining the above recurrence relations, we get the following differential equation satisfied by $C_{n}(x)$ :

$$
\left(x \frac{d^{2}}{d x^{2}}+(n+1) \frac{d}{d x}+1\right) C_{n}(x)=0 .
$$

Equation (1.6) ensures that $C_{n}(x)$ are eigen functions of the operator

$$
\mathcal{O}_{n}(x)=-\frac{d}{d x} x \frac{d}{d x}-n \frac{d}{d x} .
$$

The above operator, whose importance has been recognized within the frame work of theory of monomiality of Laguerre polynomials [2], can be viewed as a generalization of the ordinary derivative so that $C_{n}(x)$ can be considered as a generalization of the ordinary exponential function, it does not possess the semigroup property and indeed $C_{m}(x+y) \neq C_{m}(x) C_{m}(y)$. This fact far from being a limitation, allows the possibility of introducing other families of Bessel like functions.

The study of the properties of multi-variable generalized special functions has provided new means of analysis for the solutions of large classes of partial differential equations often encountered in physical problems. The relevance of the special functions in physics is well established. Most of the special functions of mathematical physics as well as their generalizations have been suggested by physical problems.

In order to further stress the usefulness of the generalized special functions, Dattoli et al. [3] have introduced the three variable two parameter extension of Tricomi functions defined as:

$$
C_{n}\left(x, y, z ; \tau_{1}, \tau_{2}\right)=\sum_{l=-\infty}^{\infty} \tau_{2}^{l} C_{n-3 l}\left(x, y ; \tau_{1}\right) C_{l}(z) .
$$

The generating function for 3-variable 2-parameter Tricomi functions $(3 \mathrm{~V} 2 \mathrm{PTF}) C_{n}\left(x, y, z ; \tau_{1}, \tau_{2}\right)$ is given as:

$$
\exp \left(t-\frac{x}{t}+t^{2} \tau_{1}-\frac{y}{t^{2} \tau_{1}}+t^{3} \tau_{2}-\frac{z}{t^{3} \tau_{2}}\right)=\sum_{n=-\infty}^{\infty} t^{n} C_{n}\left(x, y, z ; \tau_{1}, \tau_{2}\right) .
$$

The $3 \mathrm{~V} 2 \mathrm{PTF} C_{n}\left(x, y, z ; \tau_{1}, \tau_{2}\right)$ are related to the 3 -variable 2-parameter Bessel functions $(3 \mathrm{~V} 2 \mathrm{PBF}) J_{n}\left(x, y, z ; \tau_{1}, \tau_{2}\right)$ by [3]

$$
C_{n}\left(x, y, z ; \tau_{1}, \tau_{2}\right)=x^{-n / 2} J_{n}\left(2 \sqrt{x}, 2 \sqrt{y}, 2 \sqrt{z} ; \frac{x}{\sqrt{y}} \tau_{1}, \tau_{2} \sqrt{\frac{x^{3}}{z}}\right)
$$


or

$$
J_{n}\left(x, y, z ; \tau_{1}, \tau_{2}\right)=\left(\frac{x}{2}\right)^{n} C_{n}\left(\frac{x^{2}}{4}, \frac{y^{2}}{4}, \frac{z^{2}}{4} ; \frac{2 y}{x^{2}} \tau_{1}, \frac{4 z}{x^{3}} \tau_{2}\right)
$$

where $J_{n}\left(x, y, z ; \tau_{1}, \tau_{2}\right)$ is given by [3]

$$
J_{n}\left(x, y, z ; \tau_{1}, \tau_{2}\right)=\sum_{l=-\infty}^{\infty} \tau_{2}^{l} J_{n-3 l}\left(x, y ; \tau_{1}\right) J_{l}(z)
$$

with the generating function

$$
\begin{aligned}
& \sum_{n=-\infty}^{\infty} J_{n}\left(x, y, z ; \tau_{1}, \tau_{2}\right) t^{n} \\
= & \exp \left(\frac{x}{2}\left(t-\frac{1}{t}\right)+\frac{y}{2}\left(t^{2} \tau_{1}-\frac{1}{t^{2} \tau_{1}}\right)+\frac{z}{2}\left(t^{3} \tau_{2}-\frac{1}{t^{3} \tau_{2}}\right)\right) .
\end{aligned}
$$

The theory of special functions from the group-theoretic point of view is a well established topic, providing a unifying formalism to deal with the immense aggregate of the special functions and a collection of formulae such as the relevant differential equations, integral representations, recurrence formulae, composition theorems, etc., see for example [13, 14]. The first significant advance in the direction of obtaining generating relations by Lie-theoretic method is made by Weisner [15-17] and Miller [10].

Within the group-theoretic context, indeed a given class of special functions appears as a set of matrix elements of irreducible representation of a given Lie group. The algebraic properties of the group are then reflected in the functional and differential equations satisfied by a given family of special functions, whilst the geometry of the homogeneous space determines the nature of the integral representation associated with the family.

Recently some contributions related to Lie-theoretical representations of generalized Laguerre and Hermite polynomials and Bessel functions of two variables have been given, see for example Khan [7], Khan and Pathan [8] and Khan et al. [9].

The 3-dimensional complex local Lie group $T_{3}$ is the set of all $4 \times 4$ matrices of the form

$$
g=\left(\begin{array}{cccc}
1 & 0 & 0 & \tau \\
0 & e^{-\tau} & 0 & c \\
0 & 0 & e^{\tau} & b \\
0 & 0 & 0 & 1
\end{array}\right), b, c, \tau \in \mathbb{C}
$$


A basis for the Lie algebra $\mathcal{T}_{3}=L\left(T_{3}\right)$ is provided by the matrices $(1.15)$

$$
\mathcal{J}^{+}=\left(\begin{array}{llll}
0 & 0 & 0 & 0 \\
0 & 0 & 0 & 0 \\
0 & 0 & 0 & 1 \\
0 & 0 & 0 & 0
\end{array}\right), \mathcal{J}^{-}=\left(\begin{array}{llll}
0 & 0 & 0 & 0 \\
0 & 0 & 0 & 1 \\
0 & 0 & 0 & 0 \\
0 & 0 & 0 & 0
\end{array}\right), \mathcal{J}^{3}=\left(\begin{array}{cccc}
0 & 0 & 0 & 1 \\
0 & -1 & 0 & 0 \\
0 & 0 & 1 & 0 \\
0 & 0 & 0 & 0
\end{array}\right)
$$

with commutation relations

$$
\left[\mathcal{J}^{3}, \mathcal{J}^{+}\right]=\mathcal{J}^{+},\left[\mathcal{J}^{3}, \mathcal{J}^{-}\right]=-\mathcal{J}^{-},\left[\mathcal{J}^{+}, \mathcal{J}^{-}\right]=0 .
$$

Also, the Lie algebra $\mathcal{E}_{3}$ of the Euclidean group $E_{3}$ (real 3-parameter global Lie group) has basis elements

$$
\mathcal{J}_{1}=\left(\begin{array}{lll}
0 & 0 & 1 \\
0 & 0 & 0 \\
0 & 0 & 0
\end{array}\right), \mathcal{J}_{2}=\left(\begin{array}{lll}
0 & 0 & 0 \\
0 & 0 & 1 \\
0 & 0 & 0
\end{array}\right), \mathcal{J}_{3}=\left(\begin{array}{ccc}
0 & -1 & 0 \\
1 & 0 & 0 \\
0 & 0 & 0
\end{array}\right),
$$

with commutation relations

$$
\left[\mathcal{J}_{1}, \mathcal{J}_{2}\right]=0,\left[\mathcal{J}_{3}, \mathcal{J}_{1}\right]=\mathcal{J}_{2},\left[\mathcal{J}_{3}, \mathcal{J}_{2}\right]=-\mathcal{J}_{1}
$$

Further, we observe that the complex matrices

$$
\mathcal{J}^{+^{\prime}}=-\mathcal{J}_{2}+i \mathcal{J}_{1}, \mathcal{J}^{-^{\prime}}=\mathcal{J}_{2}+i \mathcal{J}_{1}, \mathcal{J}^{3^{\prime}}=i \mathcal{J}_{3},(i=\sqrt{-1}),
$$

satisfy the commutation relations identical with (1.16). Thus we say that $\mathcal{T}_{3}$ is the complexification of $\mathcal{E}_{3}$ and $\mathcal{E}_{3}$ is a real form of $\mathcal{T}_{3}$ [6]. Due to this relationship between $\mathcal{T}_{3}$ and $\mathcal{E}_{3}$, the abstract irreducible representation $Q\left(\omega, m_{0}\right)$ of $\mathcal{T}_{3}[10]$ induces an irreducible representation of $\mathcal{E}_{3}$.

In this paper, we derive generating relations involving $3 \mathrm{~V} 2 \mathrm{PTF} C_{n}(x, y, z$; $\left.\tau_{1}, \tau_{2}\right)$ by using Lie-algebraic methods. In Section 2 , we give a review of the basic properties of $3 \mathrm{~V} 2 \mathrm{PTF} C_{n}\left(x, y, z ; \tau_{1}, \tau_{2}\right)$ and their special cases. In Section 3 , we derive generating relations involving $3 \mathrm{~V} 2 \mathrm{PTF}$ by using the representation $Q\left(\omega, m_{0}\right)$ of the Lie algebra $\mathcal{T}_{3}$. In Section 4 , we obtain certain new and known generating relations involving various forms of Tricomi and Bessel functions. Finally, in Section 5, some concluding remarks are given.

\section{Properties and special cases of $3 \mathrm{~V} 2 \mathrm{PTF} C_{n}\left(x, y, z ; \tau_{1}, \tau_{2}\right)$}

The 3V2PTF $C_{n}\left(x, y, z ; \tau_{1}, \tau_{2}\right)$ defined by Eqs. (1.8), (1.9) satisfy the following differential and pure recurrence relations:

$$
\begin{aligned}
\frac{\partial}{\partial x} C_{n}\left(x, y, z ; \tau_{1}, \tau_{2}\right) & =-C_{n+1}\left(x, y, z ; \tau_{1}, \tau_{2}\right), \\
\frac{\partial}{\partial y} C_{n}\left(x, y, z ; \tau_{1}, \tau_{2}\right) & =-\frac{1}{\tau_{1}} C_{n+2}\left(x, y, z ; \tau_{1}, \tau_{2}\right), \\
\frac{\partial}{\partial z} C_{n}\left(x, y, z ; \tau_{1}, \tau_{2}\right) & =-\frac{1}{\tau_{2}} C_{n+3}\left(x, y, z ; \tau_{1}, \tau_{2}\right),
\end{aligned}
$$




$$
\begin{aligned}
& \frac{\partial}{\partial \tau_{1}} C_{n}\left(x, y, z ; \tau_{1}, \tau_{2}\right)=C_{n-2}\left(x, y, z ; \tau_{1}, \tau_{2}\right)+\frac{y}{\tau_{1}^{2}} C_{n+2}\left(x, y, z ; \tau_{1}, \tau_{2}\right), \\
& \frac{\partial}{\partial \tau_{2}} C_{n}\left(x, y, z ; \tau_{1}, \tau_{2}\right)=C_{n-3}\left(x, y, z ; \tau_{1}, \tau_{2}\right)+\frac{z}{\tau_{2}^{2}} C_{n+3}\left(x, y, z ; \tau_{1}, \tau_{2}\right)
\end{aligned}
$$

and

(2.2) $n C_{n}\left(x, y, z ; \tau_{1}, \tau_{2}\right)=C_{n-1}\left(x, y, z ; \tau_{1}, \tau_{2}\right)+x C_{n+1}\left(x, y, z ; \tau_{1}, \tau_{2}\right)$

$$
\begin{aligned}
& +2 \tau_{1} C_{n-2}\left(x, y, z ; \tau_{1}, \tau_{2}\right) \\
& +3 \tau_{2} C_{n-3}\left(x, y, z ; \tau_{1}, \tau_{2}\right)+\frac{3 z}{\tau_{2}} C_{n+3}\left(x, y, z ; \tau_{1}, \tau_{2}\right) .
\end{aligned}
$$

The differential equation satisfied by $3 \mathrm{~V} 2 \mathrm{PTF} C_{n}\left(x, y, z ; \tau_{1}, \tau_{2}\right)$ is (2.3)

$$
\left(-x \frac{\partial^{2}}{\partial x^{2}}-(1+n) \frac{\partial}{\partial x}+2 \tau_{1} \frac{\partial^{2}}{\partial x \partial \tau_{1}}+3 \tau_{2} \frac{\partial^{2}}{\partial x \partial \tau_{2}}-1\right) C_{n}\left(x, y, z ; \tau_{1}, \tau_{2}\right)=0 .
$$

We note the following special cases of $3 \mathrm{~V} 2 \mathrm{PTF} C_{n}\left(x, y, z ; \tau_{1}, \tau_{2}\right)$ :

(1)

$$
C_{n}\left(\frac{x^{2}}{4}, \frac{y^{2}}{4}, \frac{z^{2}}{4} ; \frac{2 y \tau_{1}}{x^{2}}, \frac{3 z \tau_{2}}{x^{3}}\right)=\left(\frac{x}{2}\right)^{-n} J_{n}\left(x, y, z ; \tau_{1}, \tau_{2}\right),
$$

where $J_{n}\left(x, y, z ; \tau_{1}, \tau_{2}\right)$ is given by Eqs. (1.12), (1.13).

(2)

$$
C_{n}\left(\frac{x^{2}}{4}, \frac{y^{2}}{4}, \frac{z^{2}}{4} ; \frac{2 y}{x^{2}}, \frac{4 z}{x^{3}}\right)=\left(\frac{x}{2}\right)^{-n} J_{n}(x, y, z),
$$

where $J_{n}(x, y, z)$ denotes 3 -variable Bessel function (3VBF) defined by the generating function [4]

$$
\sum_{n=-\infty}^{\infty} J_{n}(x, y, z) t^{n}=\exp \left(\frac{x}{2}\left(t-\frac{1}{t}\right)+\frac{y}{2}\left(t^{2}-\frac{1}{t^{2}}\right)+\frac{z}{2}\left(t^{3}-\frac{1}{t^{3}}\right)\right) .
$$

(3)

$$
C_{n}(x, y, z ; 1,1)=C_{n}(x, y, z),
$$

where $C_{n}(x, y, z)$ denotes 3 -variable Tricomi function (3VTF) defined by the generating function

$$
\sum_{n=-\infty}^{\infty} C_{n}(x, y, z) t^{n}=\exp \left(t-\frac{x}{t}+t^{2}-\frac{y}{t^{2}}+t^{3}-\frac{z}{t^{3}}\right) .
$$

$$
C_{n}\left(x, y, z \tau_{2}^{2} ; \tau_{1} \rightarrow \tau, \tau_{2} \rightarrow 0\right)=C_{n}(x, y ; \tau)
$$


where $C_{n}(x, y ; \tau)$ denotes 2-variable 1-parameter Tricomi function (2V1PTF) defined by the generating function ([3]; p. 221, Eq.(9)).

$$
\sum_{n=-\infty}^{\infty} C_{n}(x, y ; \tau) t^{n}=\exp \left(t-\frac{x}{t}+\tau t^{2}-\frac{y}{\tau t^{2}}\right) .
$$

$$
C_{n}\left(\frac{x^{2}}{4}, \frac{y^{2}}{4}, z \tau_{2}^{2} ; \frac{2 y \tau}{x^{2}}, \tau_{2} \rightarrow 0\right)=\left(\frac{x}{2}\right)^{-n} J_{n}(x, y ; \tau),
$$

where $J_{n}(x, y ; \tau)$ denotes 2-variable 1-parameter Bessel functions (2V1PBF) defined by the generating function ([4]; p. 176 Eq.(1.2))

$$
\sum_{n=-\infty}^{\infty} J_{n}(x, y ; \tau) t^{n}=\exp \left(\frac{x}{2}\left(t-\frac{1}{t}\right)+\frac{y}{2}\left(\tau t^{2}-\frac{1}{\tau t^{2}}\right)\right) .
$$

(6)

$$
C_{n}\left(\frac{x^{2}}{4}, \frac{y^{2}}{4}, z \tau_{2}^{2} ; \frac{2 y}{x^{2}}, \tau_{2} \rightarrow 0\right)=\left(\frac{x}{2}\right)^{-n} J_{n}(x, y),
$$

where $J_{n}(x, y)$ denotes 2-variable Bessel functions (2VBF) defined by the generating function ([5]; p. 24 Eq.(1.8(a)))

$$
\sum_{n=-\infty}^{\infty} J_{n}(x, y) t^{n}=\exp \left(\frac{x}{2}\left(t-\frac{1}{t}\right)+\frac{y}{2}\left(t^{2}-\frac{1}{t^{2}}\right)\right) .
$$

$$
C_{n}\left(x, y, z \tau_{2}^{2} ; 1, \tau_{2} \rightarrow 0\right)=C_{n}(x, y),
$$

where $C_{n}(x, y)$ denotes 2-variable Tricomi functions (2VTF) defined by the generating function

$$
\sum_{n=-\infty}^{\infty} C_{n}(x, y) t^{n}=\exp \left(t-\frac{x}{t}+t^{2}-\frac{y}{t^{2}}\right)
$$

(8)

$$
C_{n}\left(\frac{x^{2}}{4}, y \tau_{1}^{2}, z \tau_{2}^{2} ; \tau_{1} \rightarrow 0, \tau_{2} \rightarrow 0\right)=\left(\frac{x}{2}\right)^{-n} J_{n}(x),
$$

where $J_{n}(x)$ denotes ordinary Bessel function.

(9)

$$
C_{n}\left(x, y \tau_{1}^{2}, z \tau_{2}^{2} ; \tau_{1} \rightarrow 0, \tau_{2} \rightarrow 0\right)=C_{n}(x),
$$

where $C_{n}(x)$ denotes Tricomi function defined by the generating function (1.4). 


\section{Generating relations}

We derive the generating relations involving $3 \mathrm{~V} 2 \mathrm{PTF} C_{n}\left(x, y, z ; \tau_{1} \tau_{2}\right)$ by framing them into the context of the representation $Q\left(\omega, m_{0}\right)$ of the Lie algebra $\mathcal{T}_{3}$. We recall that Miller [10] have determined realizations of the irreducible representation $Q\left(\omega, m_{0}\right)$ of $\mathcal{T}_{3}$ where $\omega, m_{0} \in \mathbb{C}$ such that $\omega \neq 0$ and $0 \leq$ Re $m_{0}<1$. The spectrum $S$ of this representation is the set $\left\{m_{0}+k: k\right.$ an integer $\}$ and the representation space $V$ has a basis $\left(f_{m}\right)_{m \in S}$, such that

$$
\begin{aligned}
J^{3} f_{m} & =m f_{m}, J^{+} f_{m}=\omega f_{m+1}, J^{-} f_{m}=\omega f_{m-1}, \\
C_{0,0} f_{m} & =\left(J^{+} J^{-}\right) f_{m}=\omega^{2} f_{m}, \omega \neq 0 .
\end{aligned}
$$

The commutation relations satisfied by the operators $J^{3}, J^{ \pm}$are

$$
\left[J^{3}, J^{+}\right]=J^{+},\left[J^{3}, J^{-}\right]=-J^{-},\left[J^{+}, J^{-}\right]=0 .
$$

In order to find the realizations of this representation on spaces of functions of two complex variables $x$ and $y$, Miller ([10]; pp. 59-60) has taken the functions $f_{m}(x, y)=Z_{m}(x) e^{m y}$, such that relations (3.1) are satisfied for all $m \in S$, where the differential operators $J^{3}, J^{ \pm}$are given by

$$
\begin{aligned}
J^{3} & =\frac{\partial}{\partial y} \\
J^{+} & =e^{y}\left[\frac{\partial}{\partial x}-\frac{1}{x} \frac{\partial}{\partial y}\right], \\
J^{-} & =e^{-y}\left[-\frac{\partial}{\partial x}-\frac{1}{x} \frac{\partial}{\partial y}\right] .
\end{aligned}
$$

In particular, we look for the functions

$$
f_{m}\left(x, y, z, t ; \tau_{1}, \tau_{2}\right)=Z_{m}\left(x, y, z ; \tau_{1}, \tau_{2}\right) t^{m},
$$

such that

$$
\begin{aligned}
K^{3} f_{m} & =m f_{m}, K^{+} f_{m}=\omega f_{m+1}, K^{-} f_{m}=\omega f_{m-1}, \\
C_{0,0} f_{m} & =\left(K^{+} K^{-}\right) f_{m}=\omega^{2} f_{m},(\omega \neq 0 ; m \in S) .
\end{aligned}
$$

The set of operators $\left\{K^{3}, K^{+}, K^{-}\right\}$satisfy the commutation relations identical to (3.2).

There are numerous possible solutions of Eq. (3.5). We assume that the set of linear differential operators $\left\{K^{3}, K^{+}, K^{-}\right\}$takes the form

$$
\begin{aligned}
K^{3} & =t \frac{\partial}{\partial t}, \\
K^{+} & =t \frac{\partial}{\partial x}, \\
K^{-} & =-\frac{x}{t} \frac{\partial}{\partial x}+\frac{2 \tau_{1}}{t} \frac{\partial}{\partial \tau_{1}}+\frac{3 \tau_{2}}{t} \frac{\partial}{\partial \tau_{2}}-\frac{\partial}{\partial t} .
\end{aligned}
$$

The operators in Eqs. (3.6) satisfy the commutation relations (3.2). 
In terms of the functions $Z_{m}\left(x, y, z ; \tau_{1}, \tau_{2}\right)$ and using operators (3.6), relations (3.5) reduce to

(i)

$$
\frac{\partial}{\partial x} Z_{m}\left(x, y, z ; \tau_{1}, \tau_{2}\right)=\omega Z_{m+1}\left(x, y, z ; \tau_{1}, \tau_{2}\right)
$$

(ii)

$\left[-x \frac{\partial}{\partial x}+2 \tau_{1} \frac{\partial}{\partial \tau_{1}}+3 \tau_{2} \frac{\partial}{\partial \tau_{2}}-m\right] Z_{m}\left(x, y, z ; \tau_{1}, \tau_{2}\right)=\omega Z_{m-1}\left(x, y, z ; \tau_{1}, \tau_{2}\right)$

(iii)

$$
\begin{aligned}
& {\left[-x \frac{\partial^{2}}{\partial x^{2}}+2 \tau_{1} \frac{\partial^{2}}{\partial x \partial \tau_{1}}+3 \tau_{2} \frac{\partial^{2}}{\partial x \partial \tau_{2}}-(m+1) \frac{\partial}{\partial x}\right] Z_{m}\left(x, y, z ; \tau_{1}, \tau_{2}\right) } \\
= & \omega^{2} Z_{m}\left(x, y, z ; \tau_{1}, \tau_{2}\right) .
\end{aligned}
$$

We can take $\omega=-1$, without any loss of generality. For this choice of $\omega$ and in terms of the functions $Z_{m}(x)$, relations (3.1) become ([10]; p. 60 (3.25))

(i)

(ii)

$$
\left[\frac{d}{d x}-\frac{m}{x}\right] Z_{m}(x)=-Z_{m+1}(x)
$$

$$
\left[\frac{d}{d x}+\frac{m}{x}\right] Z_{m}(x)=Z_{m-1}(x)
$$

$$
\left[-\frac{d^{2}}{d x^{2}}-\frac{1}{x} \frac{d}{d x}+\frac{m^{2}}{x^{2}}\right] Z_{m}(x)=Z_{m}(x) .
$$

We observe that (i) and (ii) of Eqs. (3.8) agree with the conventional recurrence relations for Bessel functions $J_{m}(x)$ and (iii) coincides with the differential equation for $J_{m}(x)$. Thus we see that $Z_{m}(x)=J_{m}(x)$ is a solution of Eqs. (3.8) for all $m \in S$.

Similarly, we see that for $\omega=-1$, (iii) of Eqs. (3.7) coincides with the differential equation (2.3) of $3 \mathrm{~V} 2 \mathrm{PTF} C_{n}\left(x, y, z ; \tau_{1}, \tau_{2}\right)$. In fact, for all $m \in S$ the choice for $Z_{m}\left(x, y, z ; \tau_{1}, \tau_{2}\right)=C_{m}\left(x, y, z ; \tau_{1}, \tau_{2}\right)$ satisfy Eqs. (3.7). Thus we conclude that the functions $f_{m}\left(x, y, z, t ; \tau_{1}, \tau_{2}\right)=C_{m}\left(x, y, z ; \tau_{1}, \tau_{2}\right) t^{m}, m \in S$ form a basis for a realization of the representation $Q\left(-1, m_{0}\right)$ of $\mathcal{T}_{3}$. By using ([10]; p. 18 (Theorem 1.10)), this representation of $\mathcal{T}_{3}$ can be extended to a local multiplier representation $T$ ([10], p. 17) of $T_{3}$. Using operators (3.6), the local multiplier representation $T(g), g \in T_{3}$ defined on $\mathcal{F}$, the space of all functions analytic in a neighbourhood of the point $\left(x^{0}, y^{0}, z^{0}, t^{0}, \tau_{1}^{0}, \tau_{2}^{0}\right)=(1,0,0,1,1,1)$, takes the form

$$
\left[T\left(\exp b \mathcal{J}^{+}\right) f\right]\left(x, y, z, t ; \tau_{1}, \tau_{2}\right)=f\left(x\left(1+\frac{b t}{x}\right), y, z, t ; \tau_{1}, \tau_{2}\right)
$$




$$
\begin{aligned}
{\left[T\left(\exp c \mathcal{J}^{-}\right) f\right]\left(x, y, z, t ; \tau_{1}, \tau_{2}\right)=} & f\left(x\left(1-\frac{c}{t}\right), y, z, t\left(1-\frac{c}{t}\right)\right. \\
& \left.\tau_{1}\left(1-\frac{c}{t}\right)^{-2}, \tau_{2}\left(1-\frac{c}{t}\right)^{-3}\right), \\
{\left[T\left(\exp a \mathcal{J}^{3}\right) f\right]\left(x, y, z, t ; \tau_{1}, \tau_{2}\right)=} & f\left(x, y, z, t e^{a} ; \tau_{1}, \tau_{2}\right) .
\end{aligned}
$$

If $g \in T_{3}$ is given by Eq. (1.14), we find

$$
T(g)=T\left(\exp b \mathcal{J}^{+}\right) T\left(\exp c \mathcal{J}^{-}\right) T\left(\exp a \mathcal{J}^{3}\right)
$$

and therefore we obtain

$$
\begin{aligned}
{[T(g) f]\left(x, y, z, t ; \tau_{1}, \tau_{2}\right)=} & f\left(x\left(1+\frac{b t}{x}\right)\left(1-\frac{c}{t}\right), y, z, t e^{a}\left(1-\frac{c}{t}\right)\right. \\
& \left.\tau_{1}\left(1-\frac{c}{t}\right)^{-2}, \tau_{2}\left(1-\frac{c}{t}\right)^{-3}\right),\left|\frac{b t}{x}\right|<1,\left|\frac{c}{t}\right|<1 .
\end{aligned}
$$

The matrix elements of $T(g)$ with respect to the analytic basis $\left(f_{m}\right)_{m \in S}$ are the functions $A_{l k}(g)$ uniquely determined by $Q\left(-1, m_{0}\right)$ of $\mathcal{T}_{3}$ and are defined by

$$
\begin{aligned}
& {\left[T(g) f_{m_{0}+k}\right]\left(x, y, z, t ; \tau_{1}, \tau_{2}\right) } \\
= & \sum_{l=-\infty}^{\infty} A_{l k}(g) f_{m_{0}+l}\left(x, y, z, t ; \tau_{1}, \tau_{2}\right), k=0, \pm 1, \pm 2, \pm 3, \ldots .
\end{aligned}
$$

Therefore, we prove the following result:

Theorem 3.1. The following generating equation holds

$$
\begin{aligned}
& \left(1-\frac{c}{t}\right)^{m} C_{m}\left(x\left(1+\frac{b t}{x}\right)\left(1-\frac{c}{t}\right), y, z ; \tau_{1}\left(1-\frac{c}{t}\right)^{-2}, \tau_{2}\left(1-\frac{c}{t}\right)^{-3}\right) \\
= & \sum_{p=-\infty}^{\infty} \frac{(-1)^{|p|}}{|p| !} b^{(p+|p|) / 2} c^{(-p+|p|) / 2}{ }_{0} F_{1}[-;|p|+1 ; b c] C_{m+p}\left(x, y, z ; \tau_{1}, \tau_{2}\right) t^{p}, \\
& \left|\frac{b t}{x}\right|<1,\left|\frac{c}{t}\right|<1 .
\end{aligned}
$$

Proof. Using (3.10), we obtain

$$
\begin{aligned}
& \exp (m \tau)\left(1-\frac{c}{t}\right)^{m} C_{m}\left(x\left(1+\frac{b t}{x}\right)\left(1-\frac{c}{t}\right), y, z ; \tau_{1}\left(1-\frac{c}{t}\right)^{-2}, \tau_{2}\left(1-\frac{c}{t}\right)^{-3}\right) \\
= & \sum_{l=-\infty}^{\infty} A_{l, m-m_{0}}(g) C_{m_{0}+l}\left(x, y, z ; \tau_{1}, \tau_{2}\right) t^{m_{0}+l-m}
\end{aligned}
$$


and the matrix elements $A_{l k}(g)$ are given by ([10]; p. $\left.56(3.12)^{\prime}\right)$,

$A_{l k}(g)=\exp \left(\left(m_{0}+k\right) a\right) \frac{(-1)^{|k-l|}}{|k-l| !} b^{(l-k+|k-l|) / 2} c^{(k-l+|k-l|) / 2}{ }_{0} F_{1}[-;|k-l|+1 ; b c]$,

valid for all integral values of $l, k$ and where ${ }_{0} F_{1}$ denotes confluent hypergeometric function [1].

Substituting the value of $A_{l k}(g)$ given by (3.14) into (3.13) and simplifying we obtain result (3.12).

Corollary 3.1. The following generating equation holds

$$
\begin{aligned}
& \left(1+\frac{r}{2 \nu t}\right)^{m} C_{m}\left(x\left(1+\frac{r \nu t}{2 x}\right)\left(1+\frac{r}{2 \nu t}\right), y, z ; \tau_{1}\left(1+\frac{r}{2 \nu t}\right)^{-2}, \tau_{2}\left(1+\frac{r}{2 \nu t}\right)^{-3}\right) \\
= & \sum_{p=-\infty}^{\infty}(-\nu)^{p} J_{p}(r) C_{m+p}\left(x, y, z ; \tau_{1}, \tau_{2}\right) t^{p},\left|\frac{r \nu t}{2 x}\right|<1,\left|\frac{r}{2 \nu t}\right|<1 .
\end{aligned}
$$

Proof. If $b c \neq 0$, we can introduce the co-ordinates $r, \nu$ such that $b=\frac{r \nu}{2}$ and $c=-\left(\frac{r}{2 \nu}\right)$, with these new co-ordinates the matrix elements (3.14) can be expressed as

$$
A_{l k}(g)=\exp \left(\left(m_{0}+k\right) a\right)(-\nu)^{l-k} J_{l-k}(r), k=0, \pm 1, \pm 2, \ldots .
$$

and generating relation (3.12) yields (3.15).

\section{Applications}

We discuss some applications of the generating relations obtained in the preceding section.

I. Taking $c=0$ and $t=1$ in generating relation (3.12), we get

$$
C_{m}\left((x+b), y, z ; \tau_{1}, \tau_{2}\right)=\sum_{p=0}^{\infty} \frac{(-b)^{p}}{p !} C_{m+p}\left(x, y, z ; \tau_{1}, \tau_{2}\right),\left|\frac{b}{x}\right|<1 .
$$

Again, taking $b=0$ and $t=1$ in generating relation (3.12), we get

$$
\begin{aligned}
& (1-c)^{m} C_{m}\left(x(1-c), y, z ; \tau_{1}(1-c)^{-2}, \tau_{2}(1-c)^{-3}\right) \\
= & \sum_{p=0}^{\infty} \frac{c^{p}}{p !} C_{m-p}\left(x, y, z ; \tau_{1}, \tau_{2}\right),|c|<1 .
\end{aligned}
$$

II. Replacing $x$ by $\frac{x^{2}}{4}, y$ by $\frac{y^{2}}{4}, z$ by $\frac{z^{2}}{4}, \tau_{1}$ by $\left(\frac{2 y}{x^{2}}\right) \tau_{1}, \tau_{2}$ by $\left(\frac{3 z}{x^{3}}\right) \tau_{2}$ and $t$ by $\frac{x t}{2}$ in generating relation (3.12) and using Eq. (2.4), we get

$$
\left(\frac{1-(2 c / x t)}{1+(2 b t / x)}\right)^{m / 2} J_{m}\left(x\left(1+\frac{2 b t}{x}\right)^{1 / 2}\left(1-\frac{2 c}{x t}\right)^{1 / 2}, y, z\right.
$$




$$
\begin{aligned}
& \left.\tau_{1}\left(1+\frac{2 b t}{x}\right)\left(1-\frac{2 c}{x t}\right)^{-1}, \tau_{2}\left(1+\frac{2 b t}{x}\right)^{3 / 2}\left(1-\frac{2 c}{x t}\right)^{-3 / 2}\right) \\
= & \sum_{p=-\infty}^{\infty} \frac{(-1)^{|p|}}{|p| !} b^{(p+|p|) / 2} c^{(-p+|p|) / 2}{ }_{0} F_{1}[-;|p|+1 ; b c] J_{m+p}\left(x, y, z ; \tau_{1}, \tau_{2}\right) t^{p}, \\
& \left|\frac{2 b t}{x}\right|<1,\left|\frac{2 c}{x t}\right|<1,
\end{aligned}
$$

where $J_{m}\left(x, y, z ; \tau_{1}, \tau_{2}\right)$ denotes the $3 \mathrm{~V} 2 \mathrm{PBF}$ defined by Eqs. (1.12) and (1.13). Similarly replacing $x$ by $\frac{x^{2}}{4}, y$ by $\frac{y^{2}}{4}, z$ by $\frac{z^{2}}{4}, \tau_{1}$ by $\left(\frac{2 y}{x^{2}}\right) \tau_{1}, \tau_{2}$ by $\left(\frac{3 z}{x^{3}}\right) \tau_{2}$ and $t$ by $\frac{x t}{2}$ in generating relation (3.15) and using Eq. (2.4), we get

$$
\begin{aligned}
& \left(\frac{1+(r / v x t)}{1+(r v t / x)}\right)^{m / 2} J_{m}\left(x\left(1+\frac{r v t}{x}\right)^{1 / 2}\left(1+\frac{r}{v x t}\right)^{1 / 2}, y, z ;\right. \\
& \left.\tau_{1}\left(1+\frac{r v t}{x}\right)\left(1+\frac{r}{v x t}\right)^{-1}, \tau_{2}\left(1+\frac{r v t}{x}\right)^{3 / 2}\left(1+\frac{r}{v x t}\right)^{-3 / 2}\right) \\
= & \sum_{p=-\infty}^{\infty}(-v)^{p} J_{p}(r) J_{m+p}\left(x, y, z ; \tau_{1}, \tau_{2}\right) t^{p},\left|\frac{r v t}{x}\right|<1,\left|\frac{r}{v x t}\right|<1 .
\end{aligned}
$$

Further, taking $t=\tau_{1}=\tau_{2}=1$ and $b=-c$ in generating relation (4.3), we get

$$
\begin{aligned}
& J_{m}\left(x\left(1-\frac{2 c}{x}\right), y, z\right) \\
= & \sum_{p=-\infty}^{\infty} \frac{(-1)^{3(|p|+p) / 2}}{|p| !} c^{(|p|)}{ }_{0} F_{1}\left[-;|p|+1 ;-c^{2}\right] J_{m+p}(x, y, z),\left|\frac{2 c}{x}\right|<1
\end{aligned}
$$

where $J_{m}(x, y, z)$ denotes $3 \mathrm{VBF}$ given by Eq. (2.6).

Similar result can be obtained from generating relation (4.4).

III. Replacing $z$ by $z \tau_{2}^{2}, \tau_{1}$ by $\tau$ and then taking $\tau_{2} \rightarrow 0$ in generating relation (3.12) and using Eq. (2.9), we get

$$
\begin{aligned}
& \left(1-\frac{c}{t}\right)^{m} C_{m}\left(x\left(1+\frac{b t}{x}\right)\left(1-\frac{c}{t}\right), y ; \tau\left(1-\frac{c}{t}\right)^{-2}\right) \\
= & \sum_{p=-\infty}^{\infty} \frac{(-1)^{|p|}}{|p| !} b^{(p+|p|) / 2} c^{(-p+|p|) / 2}{ }_{0} F_{1}[-;|p|+1 ; b c] C_{m+p}(x, y ; \tau) t^{p}, \\
& \left|\frac{b t}{x}\right|<1,\left|\frac{c}{t}\right|<1,
\end{aligned}
$$

where $C_{m}(x, y ; \tau)$ denotes $2 \mathrm{~V} 1 \mathrm{PTF}$ given by Eq. $(2.10)$. 
Further, replacing $x$ by $\frac{x^{2}}{4}, y$ by $\frac{y^{2}}{4}, \tau$ by $\frac{2 y \tau}{x^{2}}$ and $t$ by $\frac{x t}{2}$ in generating relation (4.6), we get

$$
\begin{aligned}
& \left(\frac{1-(2 c / x t)}{1+(2 b t / x)}\right)^{m / 2} J_{m}\left(x\left(1+\frac{2 b t}{x}\right)^{1 / 2}\left(1-\frac{2 c}{x t}\right)^{1 / 2}, y ; \tau\left(1+\frac{2 b t}{x}\right)\left(1-\frac{2 c}{x t}\right)^{-1}\right) \\
= & \sum_{p=-\infty}^{\infty} \frac{(-1)^{|p|}}{|p| !} b^{(p+|p|) / 2} c^{(-p+|p|) / 2}{ }_{0} F_{1}[-;|p|+1 ; b c] J_{m+p}(x, y ; \tau) t^{p}, \\
& \left|\frac{2 b t}{x}\right|<1,\left|\frac{2 c}{x t}\right|<1,
\end{aligned}
$$

where $J_{m}(x, y ; \tau)$ denotes $2 \mathrm{~V} 1 \mathrm{PBF}$ given by Eq. (2.12).

Further, taking $t=\tau=1$ and $b=-c$ in generating relation (4.7), we get

$$
\begin{aligned}
& J_{m}\left(x\left(1-\frac{2 c}{x}\right), y\right) \\
= & \sum_{p=-\infty}^{\infty} \frac{(-1)^{3(|p|+p) / 2}}{|p| !} c^{(|p|)}{ }_{0} F_{1}\left[-;|p|+1 ;-c^{2}\right] J_{m+p}(x, y),\left|\frac{2 c}{x}\right|<1,
\end{aligned}
$$

where $J_{m}(x, y)$ denotes $2 \mathrm{VBF}$ given by Eq. (2.14).

Similar results can be obtained from generating relation (3.15).

IV. Replacing $y$ by $y \tau_{1}{ }^{2}, z$ by $z \tau_{2}{ }^{2}$ and then taking $\tau_{1}, \tau_{2} \rightarrow 0$ in generating relations (3.12) and (3.15) and using Eq. (2.18), we get

$$
\begin{aligned}
& \left(1-\frac{c}{t}\right)^{m} C_{m}\left(x\left(1+\frac{b t}{x}\right)\left(1-\frac{c}{t}\right)\right) \\
= & \sum_{p=-\infty}^{\infty} \frac{(-1)^{|p|}}{|p| !} b^{(p+|p|) / 2} c^{(-p+|p|) / 2}{ }_{0} F_{1}[-;|p|+1 ; b c] C_{m+p}(x) t^{p}, \\
& \left|\frac{b t}{x}\right|<1,\left|\frac{c}{t}\right|<1
\end{aligned}
$$

and

$$
\begin{aligned}
& \left(1+\frac{r}{2 \nu t}\right)^{m} C_{m}\left(x\left(1+\frac{r \nu t}{2 x}\right)\left(1+\frac{r}{2 \nu t}\right)\right) \\
= & \sum_{p=-\infty}^{\infty}(-\nu)^{p} J_{p}(r) C_{m+p}(x) t^{p},\left|\frac{r \nu t}{2 x}\right|<1,\left|\frac{r}{2 \nu t}\right|<1,
\end{aligned}
$$

respectively, where $C_{m}(x)$ denotes Tricomi function given by Eqs. (1.1) and (1.4). 
Further, replacing $x$ by $z^{2} / 4, t$ by $z t / 2$ in generating relation (4.9) and using Eq. (1.3), we obtain ([10]; p.62(3.29)), for $\left.Z_{m}=J_{m}\right)$

$$
\begin{aligned}
& \left(\frac{\left(1-\frac{2 c}{z t}\right)}{\left(1+\frac{2 b t}{z}\right)}\right)^{m / 2} J_{m}\left(z\left(1+\frac{2 b t}{z}\right)^{1 / 2}\left(1-\frac{2 c}{z t}\right)^{1 / 2}\right) \\
= & \sum_{p=-\infty}^{\infty} \frac{(-1)^{|p|}}{|p| !} b^{(p+|p|) / 2} c^{(-p+|p|) / 2}{ }_{0} F_{1}[-;|p|+1 ; b c] J_{m+p}(z) t^{p}, \\
& \left|\frac{2 b t}{z}\right|<1,\left|\frac{2 c}{z t}\right|<1 .
\end{aligned}
$$

Several of the fundamental identities for cylindrical functions are special cases of generating relation (4.11). Also, for $c=0, t=1$ and $b=0, t=1$, relation (4.11) gives the formulas of Lommel ([10]; p. 62 (3.30) and (3.31) for $\left.Z_{m}=J_{m}\right)$.

Again, replacing $x$ by $z^{2} / 4, t$ by $z / 2$ in generating relation (4.10) and using Eq.(1.3), we obtain a generalization of Graf's addition theorem ([10]; p. $63(3.32)$, for $\left.Z_{m}=J_{m}\right)$

$$
\begin{aligned}
& \left(\frac{\left(1+\frac{r}{\nu z}\right)}{\left(1+\frac{r \nu}{z}\right)}\right)^{m / 2} J_{m}\left(z\left(1+\frac{r \nu}{z}\right)^{1 / 2}\left(1+\frac{r}{\nu z}\right)^{1 / 2}\right) \\
= & \sum_{p=-\infty}^{\infty}(-\nu)^{p} J_{p}(r) J_{m+p}(z),\left|\frac{r \nu}{z}\right|<1,\left|\frac{r}{\nu z}\right|<1 .
\end{aligned}
$$

Further, taking $c=0, t=1$ and replacing $x$ by $x^{2} / 4$ and $b$ by $-x t / 2$ in relation (4.9) and using Eq. (1.3), we get a well known generating relation ([12]; p. 427, Eq. (56)).

$$
\sum_{p=0}^{\infty} \frac{t^{p}}{p !} J_{m+p}(x)=\left(\frac{x}{x-2 t}\right)^{m / 2} J_{m}\left(\sqrt{x^{2}-2 x t}\right), m \in \mathbb{C}
$$

\section{Concluding remarks}

We note that the expressions (3.11) are valid only for group elements $g$ in a sufficiently small neighbourhood of the identity element of the Lie group $T_{3}$. However, we can also use operators (3.6) to derive generating relations for $3 \mathrm{~V} 2 \mathrm{PTF}$ and related functions with group elements bounded away from the identity.

If $f\left(x, y, z, t ; \tau_{1}, \tau_{2}\right)$ is a solution of the equation $C_{0,0} f=\omega^{2} f$, i.e.,

$$
\begin{aligned}
& \left(-x \frac{\partial^{2}}{\partial x^{2}}+2 \tau_{1} \frac{\partial^{2}}{\partial x \partial \tau_{1}}+3 \tau_{2} \frac{\partial^{2}}{\partial x \partial \tau_{2}}-(m+1) \frac{\partial}{\partial x}\right) f\left(x, y, z, t ; \tau_{1}, \tau_{2}\right) \\
= & \omega^{2} f\left(x, y, z, t ; \tau_{1}, \tau_{2}\right),
\end{aligned}
$$


then the function $T(g) f$ given by (3.10) satisfies the equation

$$
C_{0,0}(T(g) f)=\omega^{2}(T(g) f) .
$$

This follows from the fact that $C_{0,0}$ commutes with the operators $K^{+}, K^{-}$ and $K^{3}$. Now if $f$ is a solution of the equation

$$
\left(x_{1} K^{+}+x_{2} K^{-}+x_{3} K^{3}\right) f\left(x, y, z, t ; \tau_{1}, \tau_{2}\right)=\lambda f\left(x, y, z, t ; \tau_{1}, \tau_{2}\right)
$$

for constants $x_{1}, x_{2}, x_{3}$ and $\lambda$, then $T(g) f$ is a solution of the equation

$$
\left[T(g)\left(x_{1} K^{+}+x_{2} K^{-}+x_{3} K^{3}\right) T\left(g^{-1}\right)\right][T(g) f]=\lambda[T(g) f] .
$$

The inner automorphism $\mu_{g}$ of Lie group $T_{3}$ defined by

$$
\mu_{g}(h)=g h g^{-1}, h \in T_{3},
$$

induces an automorphism $\mu_{g}^{\star}$ of Lie algebra $\mathcal{T}_{3}$ where

$$
\mu_{g}^{\star}(\alpha)=g \alpha g^{-1}, \alpha \in \mathcal{T}_{3} .
$$

If $\alpha=x_{1} \mathcal{J}^{+}+x_{2} \mathcal{J}^{-}+x_{3} \mathcal{J}^{3}$, where $\mathcal{J}^{+}, \mathcal{J}^{-}$and $\mathcal{J}^{3}$ are given by Eq. (1.15) and $g$ is given by Eq. (1.14), then we have

$$
\mu_{g}^{\star}(\alpha)=\left(x_{1} e^{a}-b x_{3}\right) \mathcal{J}^{+}+\left(x_{2} e^{-a}+c x_{3}\right) \mathcal{J}^{-}+x_{3} \mathcal{J}^{3},
$$

as a consequence of which, we can write

$T(g)\left(x_{1} K^{+}+x_{2} K^{-}+x_{3} K^{3}\right) T\left(g^{-1}\right)=\left(x_{1} e^{a}-b x_{3}\right) K^{+}+\left(x_{2} e^{-a}+c x_{3}\right) K^{-}+x_{3} K^{3}$.

To give an example of the application of these remarks, we consider the function $f\left(x, y, z, t ; \tau_{1}, \tau_{2}\right)=C_{m}\left(x, y, z ; \tau_{1}, \tau_{2}\right) t^{m}, m \in \mathbb{C}$. Since $C_{0,0} f=f$ and $K^{3} f=m f$, so the function

$$
\begin{aligned}
& {[T(g) f]\left(x, y, z, t ; \tau_{1}, \tau_{2}\right) } \\
= & e^{m a}(t-c)^{m} C_{m}\left((x+b t)\left(1-\frac{c}{t}\right), y, z ; \tau_{1}\left(1-\frac{c}{t}\right)^{-2}, \tau_{2}\left(1-\frac{c}{t}\right)^{-3}\right),
\end{aligned}
$$

satisfies the equations

$$
\begin{gathered}
C_{0,0}[T(g) f]=[T(g) f], \\
\left(-b K^{+}+c K^{-}+K^{3}\right)[T(g) f]=m[T(g) f] .
\end{gathered}
$$

For $a=b=0$ and $c=-1$, we can express the function (5.7) in the form

(5.10) $h\left(x, y, z, t ; \tau_{1}, \tau_{2}\right)$

$$
=(t+1)^{m} C_{m}\left(\left(x+\frac{x}{t}\right), y, z ; \tau_{1}\left(1+\frac{1}{t}\right)^{-2}, \tau_{2}\left(1+\frac{1}{t}\right)^{-3}\right),|t|<1 .
$$

Now using the Laurent expansion

$$
h\left(x, y, z, t ; \tau_{1}, \tau_{2}\right)=\sum_{k=-\infty}^{\infty} h_{k}\left(x, y, z ; \tau_{1}, \tau_{2}\right) t^{k},|t|<1,
$$


in Eq. (5.8), we note that $h_{k}\left(x, y, z ; \tau_{1}, \tau_{2}\right)$ is a solution of differential equation (2.3) for each integer $k$. Since the function $h\left(x, y, z, t ; \tau_{1}, \tau_{2}\right)$ is bounded for $x=y=z=0$, we have

$$
h_{k}\left(x, y, z ; \tau_{1}, \tau_{2}\right)=c_{k} C_{k}\left(x, y, z ; \tau_{1}, \tau_{2}\right), c_{k} \in \mathbb{C} .
$$

Thus

$$
h_{k}\left(x, y, z, t ; \tau_{1}, \tau_{2}\right)=\sum_{k=-\infty}^{\infty} c_{k} C_{k}\left(x, y, z ; \tau_{1}, \tau_{2}\right) t^{k} .
$$

Now from Eq. (5.9), we have

$$
\left(-K^{-}+K^{3}\right) h\left(x, y, z, t ; \tau_{1}, \tau_{2}\right)=m h\left(x, y, z, t ; \tau_{1}, \tau_{2}\right)
$$

and therefore it follows that

$$
c_{k+1}=(m-k) c_{k} .
$$

Further taking $x=y=z=0$ in (5.10) and using (5.11), we get $c_{0}=$ $1 / \Gamma(m+1)$ and hence $c_{k}=1 / \Gamma(m-k+1)$. Thus we obtain the following result:

$$
\begin{aligned}
& (t+1)^{m} C_{m}\left(\left(x+\frac{x}{t}\right), y, z ; \tau_{1}\left(1+\frac{1}{t}\right)^{-2}, \tau_{2}\left(1+\frac{1}{t}\right)^{-3}\right) \\
= & \sum_{k=-\infty}^{\infty} \frac{C_{k}\left(x, y, z ; \tau_{1}, \tau_{2}\right) t^{k}}{\Gamma(m-k+1)},|t|<1,
\end{aligned}
$$

which is obviously not a special case of generating relation (3.12).

Several other examples of generating relations can be derived by this method, see for example Weisner [17].

We have considered $3 \mathrm{~V} 2 \mathrm{PTF} C_{m}\left(x, y, z ; \tau_{1}, \tau_{2}\right)$ within the group representation formalism. These functions appeared as basis functions for a realization of the representation $Q\left(-1, m_{0}\right)$ of the Lie algebra $\mathcal{T}_{3}$. The analysis presented in this paper confirms the possibility of extending this approach to other useful forms of generalized Tricomi functions as well as to their Bessel counter parts.

\section{References}

[1] L. C. Andrews, Special Functions for Engineers and Applied Mathematician, Macmillan Company, New York, 1985.

[2] G. Dattoli, Hermite-Bessel and Laguerre-Bessel functions: a by-product of the monomiality principle, Advanced special functions and applications, (Melfi, 1999), 147-164, Proc. Melfi Sch. Adv. Top. Math. Phys. 1, Aracne, Rome 2000.

[3] G. Dattoli, M. Migliorati, and H. M. Srivastava, Some families of generating functions for the Bessel and related functions, Georgian Math. J. 11 (2004), no. 2, 219-228.

[4] G. Dattoli and A. Torre, Theory and Applications of Generalized Bessel Functions, ARACNE, Rome, Italy, 1996.

[5] G. Dattoli, A. Torre, S. Lorenzutta, G. Maino, and C. Chiccoli, Theory of generalized Bessel functions. II, Nuovo Cimento B (11) 106 (1991), no. 1, 21-51. 
[6] S. Helgason, Differential Geometry and Symmetric Spaces, Academic Press, New York, 1962.

[7] S. Khan, Harmonic oscillator group and Laguerre 2D polynomials, Rep. Math. Phys. 52 (2003), 227-233.

[8] S. Khan and M. A. Pathan, Lie theoretic generating relations of Hermite $2 D$ polynomials, J. Comput. Appl. Math. 160(2003), no. 1-2, 139-146.

[9] S. Khan, G. Yasmeen, and A. Mittal, Representation of Lie algebra $\mathrm{T}_{3}$ and 2-variable 2-parameter Bessel functions, J. Math. Anal. Appl. 326 (2007), no. 1, 500-510.

[10] W. Jr. Miller, Lie Theory and Special Functions, Academic Press, New York and London, 1968.

[11] E. D. Rainville, Special Functions, Macmillan, Reprinted by Chelsea Publ. Co., Bronx, New York, 1971.

[12] H. M. Srivastava and H. L. Manocha, A Treatise on Generating Functions, Ellis Horwood Limited, Chichester, 1984.

[13] N. Ja. Vilenkin, Special Functions and the Theory of Group Representations, Amer. Math. Soc. Trans. Providence, Rhode Island, 1968.

[14] A. Wawrzyn̄czyk, Group Representation and Special Functions, PWN-Polish Scientific Publ., Warszawa, 1984.

[15] L. Weisner, Group-theoretic origin of certain generating functions, Pacific J. Math. 5 (1955), 1033-1039.

[16] _ Generating functions for Hermite functions, Canad. J. Math. 11 (1959), 141147.

[17] _ Generating functions for Bessel functions, Canad. J. Math. 11 (1959), 148-155.

SUBUHi KHAN

Department of Mathematics

Aligarh Muslim University

Aligarh-202002, India

E-mail address: subuhi2006@gmail.com

Mumtaz Ahmad Khan

Department of Applied Mathematics

FACULTY OF ENGINEERING

Aligarh Muslim University

Aligarh-202002, INDIA

Rehana Khan

Department of Applied Mathematics

FACULTY OF ENGINEERING

Aligarh Muslim University

Aligarh-202002, INDIA 